\title{
Les fusions théâtrales dans les nouveaux Länder : héritage culturel, fardeau financier
}

Theatrical fusions in East Germany

Laure de Verdalle

\section{(2) OpenEdition}

12 Journals

Édition électronique

URL : http://journals.openedition.org/travailemploi/4850

DOI : 10.4000/travailemploi.4850

ISSN : 1775-416X

Éditeur

DARES - Ministère du Travail

Édition imprimée

Date de publication : 15 octobre 2006

Pagination : 33-43

ISSN : 0224-4365

\section{Référence électronique}

Laure de Verdalle, «Les fusions théâtrales dans les nouveaux Länder : héritage culturel, fardeau financier », Travail et Emploi [En ligne], 108 | octobre-décembre 2006, mis en ligne le 15 décembre 2008, consulté le 23 avril 2019. URL : http://journals.openedition.org/travailemploi/4850 ; DOI : 10.4000/travailemploi.4850 


\title{
Les fusions théâtrales dans les nouveaux Länder
}

\author{
Héritage culturel, fardeau financier
}

Laure de Verdalle $\left(^{*}\right)$

La réunification politique des deux Allemagnes a eu de fortes incidences sur l'organisation du travail théâtral dans les nouveaux Länder. À partir d'un socle historique commun (la République de Weimar), les deux Allemagnes ont développé durant quarante années des politiques publiques théâtrales très différentes, pour ne pas dire opposées : tandis qu'un processus de rationalisation du tissu théâtral a été mis très tôt en æuvre en RFA, aboutissant à une relative concentration des lieux scéniques, la RDA s'est caractérisée au contraire par une grande densité de structures "multigenres ». Dès le début de la transition, c'est le modèle ouest-allemand qui semble imposer ses normes. Les villes moyennes des nouveaux Länder doivent regrouper à marche forcée leurs structures culturelles afin de réaliser des économies d'échelle. L'article étudie ces processus de fusion tant du point de vue matériel et politique que de celui de la production culturelle et de l'organisation du travail artistique. Mais loin de confirmer la prédominance du modèle fédéral ouest-allemand sur les structures théâtrales de l'exAllemagne de l'Est, les processus de rationalisation économique et administrative dans la sphère culturelle se traduiraient par un «effet en retour» du modèle dominé sur le modèle dominant.

En 1990, lors de la réunification des deux Allemagnes, la RDA présente un paysage théâtral public d'une exceptionnelle densité. Une soixantaine d'Ensembles se partagent 173 lieux scéniques et emploient près de 17000 personnes, toutes activités confondues. À titre de comparaison, la RFA compte alors 88 théâtres publics (pour une population quatre fois supérieure) employant 28000 personnes (1). Les nouveaux Länder se distinguent également de leurs homologues de l'Ouest par la répartition géographique de leurs institutions théâtrales publiques dont $42 \%$ sont situées dans des villes de moins de 50000 habitants, contre $7 \%$ seulement au sein des anciens Länder (2). Enfin, les théâtres publics de 1'Est sont majoritairement des maisons « multigenres » (Mehrspartentheater), qui regroupent sous un même toit théâtre, orchestre, opéra et parfois même ballet.

(*) Chargée de recherche CNRS. Laboratoire «Printemps», Guyancourt : laure.de-verdalle@printemps.uvsq.fr.

(1) À l'Est comme à l'Ouest, les théâtres publics fonctionnent selon le modèle de l'ensemble, qui réunit une troupe fixe de comédiens, metteurs en scène, chanteurs, etc. ainsi qu'un personnel technique et administratif permanent. Mais la RDA a généralisé en 1972 l'utilisation du contrat à durée indéterminée au sein de ses théâtres alors que la RFA fonctionne avec des contrats à durée déterminée d'un ou deux ans.

(2) Berlin n'est pas prise en compte dans cette comparaison.
Ces différences, qui se sont construites à partir d'un héritage commun(3), doivent être rapportées aux politiques culturelles et artistiques menées durant quarante ans par les deux États allemands. Tandis que la RFA, par des stratégies de concentration et des réformes structurelles, a entamé assez tôt la rationalisation de son tissu théâtral public, la RDA, en partie grâce à une politique culturelle fortement centralisée, a garanti la survie des institutions théâtrales publiques dispersées sur l'ensemble de son territoire.

En 1990, le tissu théâtral est-allemand se trouve brutalement confronté à un modèle de gestion publique des affaires culturelles importé de l'Ouest. Pour les théâtres de l'ex-RDA, la transition se traduit notamment par le passage d'un financement centralisé à un financement décentralisé de la culture (LABORIER, 1992). La vie culturelle est en effet un des domaines dans lesquels s'exprime le mieux le principe du fédéralisme ouest-allemand : ce sont les villes et les Länder qui supportent la charge financière de la majeure partie des équipements culturels(4). Dans

(3) Celui de la République de Weimar qui avait consolidé un modèle théâtral reposant sur une municipalisation de la culture, autour d'institutions publiques.

(4) En 1989, les dépenses culturelles de la RFA se sont élevées à 9 milliards de DM: $55 \%$ de cette somme provient des communes, $38 \%$ des Länder et seulement $7 \%$ du Bund (WIESAND, 1992). 
les anciens Länder, la taille moyenne d'une ville susceptible d'entretenir un Mehrspartentheater et son ensemble est de 200000 habitants(5). On comprend dès lors les difficultés rencontrées par les villes estallemandes pour conserver leur institution théâtrale après la réunification, et ce d'autant plus qu'elles se heurtent à d'importantes difficultés économiques et sociales. Dans ce contexte, on observe tout au long des années 1990 une progressive remise en cause du paysage théâtral est-allemand, qui prend toutefois des formes différentes en fonction des stratégies adoptées par les Länder de l'Est et par leurs municipalités (Verdalle (DE), 2002). C'est l'une de ces stratégies, la fusion théâtrale, que nous nous proposons d'étudier ici car elle incarne bien l'exigence de mise aux normes ouest-allemandes du paysage théâtral hérité de l'ex-RDA. En effet, la fusion est un instrument de rationalisation du tissu théâtral qui répond à l'introduction de nouveaux modèles de gestion des affaires culturelles. Toutefois, les cas qui seront étudiés ici sont ceux de fusions opérant entre des Ensembles est-allemands. La question traitée sera donc moins celle des difficultés du management interculturel(6) (BARMEYER, 2005) que celle de la restructuration d'un paysage théâtral et des organisations qui le composent face aux contraintes (notamment financières) introduites par la réunification.

Pour analyser les conséquences de ce choc, nous commencerons par rappeler rapidement quelques caractéristiques générales de la réunification et de ses effets en matière de politique culturelle. Puis nous étudierons différents cas de fusions théâtrales menées en Saxe et en Thuringe, en montrant qu'au-delà d'un cadre commun (la réunion de deux Ensembles publics et le partage de leurs lieux scéniques), plusieurs options ont été mobilisées, qui font intervenir des rapports de force entre les municipalités et entre les institutions théâtrales concernées. Nous pourrons alors analyser les conséquences locales de ces fusions sur l'organisation du travail théâtral, sur les modalités de la production et sur l'état des relations au public. Nous conclurons en interrogeant l'impact à moyen/long terme de ces fusions (notamment en matière de droit du travail).

(5) En ce qui concerne les trois Länder que j'ai étudiés dans le cadre de ma thèse de doctorat, seule la Saxe présente trois villes qui remplissent cette condition (Dresde, Leipzig et Chemnitz). En Thuringe, Erfurt, la capitale du Land atteint à peine ce plancher. Et dans le Brandebourg, Potsdam, la plus grande ville, compte seulement quelque 120000 habitants.

(6) Même si certaines entités issues des fusions ont été confiées à la gestion d'intendants venus de l'Ouest.

\section{Encadré 1 \\ Méthodologie}

Ce texte s'appuie sur les résultats d'une thèse de sociologie soutenue en octobre 2003 et consacrée à la recomposition du monde théâtral est-allemand après la réunification. Cette recherche visait à étudier conjointement : 1) l'action des politiques publiques sur le remodelage du tissu théâtral des nouveaux Länder; 2) les transformations organisationnelles qui s'opèrent au sein des théâtres publics durant les années 1990 ; et 3) les parcours professionnels des gens de théâtre originaires de l'ex-RDA.

En ce qui concerne plus particulièrement les fusions, l'enquête s'est concentrée sur les Länder de Saxe et de Thuringe. En effet, tous deux ont eu recours aux fusions dans les années 1990 et présentaient, au moment de la réunification, des paysages théâtraux relativement similaires et extrêmement denses. Par ailleurs, ces deux Länder se caractérisaient par la forte présence d'institutions théâtrales publiques dans des petites villes de moins de 50000 habitants (même si la Saxe compte également deux grandes villes de plus de 500000 habitants, Dresde et Leipzig).

Quatre cas de fusion ont fait l'objet d'une analyse approfondie :

\begin{tabular}{|c|c|c|c|c|}
\hline & $\begin{array}{c}\text { Altenburg/ } \\
\text { Gera } \\
\text { (Thuringe) }\end{array}$ & $\begin{array}{l}\text { Eisenach/ } \\
\text { Rudolstadt } \\
\text { (Thuringe) }\end{array}$ & $\begin{array}{c}\text { Freiberg/ } \\
\text { Döbeln } \\
\text { (Saxe) }\end{array}$ & $\begin{array}{c}\text { Plauen/ } \\
\text { Zwickau } \\
\text { (Saxe) }\end{array}$ \\
\hline $\begin{array}{l}\text { Date } \\
\text { de la fusion }\end{array}$ & 1995 & 1995 & 1993 & 2000 \\
\hline $\begin{array}{l}\text { Nombre } \\
\text { d'habitants }\end{array}$ & $\begin{array}{c}\text { Altenburg: } \\
46000 \\
\text { Gera: } \\
126000\end{array}$ & $\begin{array}{c}\text { Eisenach : } \\
46000 \\
\text { Rudolstadt : } \\
29000\end{array}$ & $\begin{array}{c}\text { Freiberg: } \\
48000 \\
\text { : Döbeln : } \\
25000\end{array}$ & $\begin{array}{r}\text { Plauen: } \\
68000 \\
\text { Zwickau } \\
105000\end{array}$ \\
\hline $\begin{array}{l}\text { Distance } \\
\text { séparant les } \\
\text { deux villes }\end{array}$ & $35 \mathrm{~km}$ & $90 \mathrm{~km}$ & $36 \mathrm{~km}$ & $42 \mathrm{~km}$ \\
\hline
\end{tabular}

Chacun de ces cas a donné lieu à une monographie, construite à partir des données du Deutsche Bühnenverein (1), antérieures et postérieures à la fusion (budgets, effectifs employés, nombre de mises en scène produites, nombre de représentations dans les différents lieux scéniques, taux de fréquentation, etc.). Des entretiens ont été conduits au sein des Ensembles, afin de revenir sur les changements organisationnels consécutifs aux fusions, mais aussi dans les ministères de la Culture ainsi que dans les services culturels des municipalités concernées, afin de saisir les enjeux locaux des fusions et les stratégies mises en œuvre par chaque Land dans la restructuration de son paysage théâtral.

(1) Cette association des scènes allemandes, créée en 1846, s'intéresse à toutes les questions de politique culturelle relatives au théâtre et à son organisation. Elle publie chaque année des statistiques détaillées. 


\section{Le passage d'une gestion centralisée à une gestion décentralisée de la culture}

Le traité d'unification, signé en août 1990, entérine l'extension du modèle fédéral et administratif ouest-allemand à la partie Est de l'Allemagne. En effet, la réalisation de l'unité allemande par le biais de l'article 23 de la Loi Fondamentale impose la réorganisation du territoire est-allemand en Länder et sa mise aux normes ouest-allemandes. La loi sur la formation des nouveaux Länder(7) prend effet le 14 octobre 1990 et ses conséquences dans le domaine des politiques culturelles sont immédiates : le ministère est-allemand de la Culture est démantelé et le traité d'unification opère un transfert des compétences en matière de gestion et de financement de la culture vers les Länder et les communes. Des ministères régionaux compétents pour les arts, la culture et l'éducation sont mis en place.

Mais si la réunification des deux Allemagnes intervient rapidement, un an à peine après la chute du mur, la renaissance de ces entités territoriales (8) et leur autonomisation suivent un processus marqué par plusieurs étapes. Dans le domaine de la culture, le Bund est amené à jouer un rôle transitoire important, contrevenant par là même à sa tradition de nonintervention dans les affaires culturelles des Länder. Le traité d'unification comprend ainsi une clause culturelle (l'article 35), qui concerne la sauvegarde de la «substance culturelle» est-allemande. Ce principe de conservation conduit le Bund à financer plusieurs programmes d'aide exceptionnels, qui atteignent un total de 900 millions de DM pour l'année 1991 et de 2,9 milliards de DM jusqu'en 1994 (LABORIER, 1996). Toutefois, ces aides se tarissent assez rapidement et à partir de 1994 le Bund se fait plus discret.

Les nouveaux Länder restent alors seuls face au modèle décentralisé de gestion des affaires culturelles et doivent définir leurs propres stratégies de financement. Pour cela, ils vont s'inspirer de ce qui existe dans les anciens Länder (le principe des partenariats administratifs entre anciens et nouveaux Länder favorise la transmission des expériences), tout en profitant de la plasticité inhérente au fédéralisme. La comparaison de deux cas, celui de la Saxe et celui de la Thuringe ( $c f$. encadrés 2 et 3 ), permet de comprendre comment les choix mis en œuvre s'inscrivent dans des situations économiques et politiques locales ainsi que dans des histoires régionales et urbaines.

(7) Saxe, Thuringe, Saxe-Anhalt, Brandebourg et Mecklembourg Poméranie Occidentale.

(8) La RDA avait supprimé les Länder en 1952.
Encadré 2

\section{La Saxe ou la défense d'une politique autonome}

Dans les années qui suivent la réunification, la Saxe exploite largement l'image d'une tradition culturelle et d'une identité locale fortes et anciennes. Assez proche en cela de la Bavière (à l'Ouest), ce Land affirme sa spécificité au sein des nouveaux Länder en inventant des mécanismes originaux permettant d'amortir la transition. La loi sur les espaces culturels saxons, adoptée en décembre 1993, vise ainsi à réorganiser les relations entre les différents niveaux d'administration locale afin d'assurer dans la durée le financement des institutions culturelles. Chacun des espaces est pensé comme une «coopérative», mettant en commun des ressources financières pour soutenir la vie culturelle.

Mais ce dynamisme est hypothéqué par la nécessaire coordination entre les différents niveaux administratifs et par la fragilité financière des communes saxonnes, qui rendent difficile la gestion du dispositif basé sur un principe de solidarité. La loi de 1993 apparaît surtout comme une tentative d'adaptation défensive du Land et des communes face aux exigences introduites par le traité d'unification. La fusion qui intervient en 2000 entre les théâtres de Plauen et de Zwickau ouvre la voie à une restructuration radicale (bien que retardée) du paysage théâtral saxon. En 2002, une nouvelle fusion réunit les théâtres de Bautzen et de Zittau et la même année, le maire de Dresde, avec le soutien du ministère de la Culture du Land, propose de fusionner l'opérette et le Staatsschauspiel, envisageant même à plus long terme de réunir toutes les scènes publiques de la ville sous un même conseil d'intendance. Ce dernier projet soulève l'opposition farouche du milieu théâtral, mais ne semble pas totalement abandonné.

La Saxe et la Thuringe ont donc l'une et l'autre recours aux fusions pour restructurer leur paysage théâtral dans les années qui suivent la réunification. Cette stratégie, présentée comme une alternative aux fermetures de théâtres, est toutefois ponctuée d'hésitations et de nombreux retournements d'alliance. Pour comprendre ces tâtonnements, regardons à présent comment ces fusions fonctionnent concrètement et quelles sont les difficultés qu'elles posent, à différents niveaux. L'analyse des conséquences de la réunification dans le domaine théâtral rejoint ici un questionnement plus large portant sur l'extension des modèles gestionnaires à la sphère culturelle. 


\section{Encadré 3 \\ La Thuringe ou la crise du modèle ouest-allemand de financement de la culture}

La Thuringe se conforme plus fidèlement au modèle ouest-allemand de financement de la culture et se heurte très vite à la question de la rationalisation d'un paysage théâtral particulièrement dense. Dès le début des années 1990, le Land a construit sa politique théâtrale à partir d'une carte, raisonnant en termes de régions et d'espaces de spectateurs (Zuschauerraum) :

«Prenons le public qui vit ici [il désigne un point sur la carte du Land, proche de la ville de Sonderhausen] : où se trouve pour lui la possibilité la plus proche d'aller au théâtre? Erfurt est à $75 \mathrm{~km}$, et aujourd'hui encore, c'est un temps de trajet de près de 45 minutes. Ou Eisenach, mais c'est à $80 \mathrm{~km}$ ». Directeur du théâtre au ministère de la Culture du Land de Thuringe, Erfurt, avril 2002.

Le principe des fusions est rapidement mis en avant par le ministère de la Culture du Land pour préserver une offre théâtrale diversifiée sur l'ensemble du territoire. Il s'agit de restructurer en profondeur un tissu théâtral fortement concentré dans des villes de petite et moyenne importance (en 1991, cinq des huit théâtres du Land se trouvent dans des villes de moins de 50000 habitants). Une première vague de fusions d'orchestres a lieu dès 1992. Une seconde vague, en 1995, fait cette fois intervenir des fusions entre théâtres (Altenburg et Gera d'une part, Rudolstadt et Eisenach de l'autre). À partir de 2000-2001, une troisième étape s'amorce, et prévoit de regrouper trois entités qui sont elles-mêmes le résultat de fusions antérieures (le théâtre d'Eisenach/Rudolstadt, celui de Nordhausen/ Sonderhausen et la philharmonie de GothaSuhl). Après de multiples tergiversations, le ministère de la Culture de Thuringe fait finalement marche arrière. En 2003, la fusion entre Eisenach et Rudolstadt (problématique depuis l'origine en raison de la distance séparant les deux villes) est cassée. Les deux théâtres retrouvent leur autonomie mais sont sommés de nouer de nouveaux partenariats : Rudolstadt semble aujourd'hui avoir accepté l'idée d'un rapprochement avec Nordhausen, tandis qu'Eisenach envisage une coopération avec Meinigen.

\section{Des négociations difficiles}

Une fusion entraine la création d'une nouvelle entité théâtrale qui se présente de la manière suivante : un seul Ensemble disposant de plusieurs lieux scéniques, dans différentes villes (deux pour commencer, les deux partenaires de la fusion). Pour parveniràcerésultat, et puisqueles théâtres concernés sont presque toujours des Mehrspartentheater, la modalité la plus fréquente est celle d'un partage des genres : l'une des villes hérite du théâtre (Schauspiel) et l'autre de l'opéra (Musiktheater). Toutes deux conservent un ensemble à demeure (les comédiens d'un côté, les solistes, l'orchestre et le chœur de l'autre), et les genres tournent ensuite d'une ville à l'autre au grès des représentations. L'objectif est de maintenir une offre théâtrale diversifiée, tout en réduisant les coûts de production qui sont partagés entre les villes partenaires de la fusion.

\section{Le poids des luttes d'influence}

Mais si les municipalités concernées par les fusions se retrouvent autour d'un objectif d'économies d'échelle, être cogestionnaire de ces nouvelles entités ne va pas de soi. Différents facteurs doivent être pris en compte : 1'histoire des villes et de leur théâtre, le rôle traditionnel du Stadttheater dans l'espace urbain en Allemagne, les vieilles rivalités opposant certaines communes, etc. Ainsi, lors de la fusion entre les théâtres de Freiberg et de Döbeln, dans la Saxe, le profil très différent des deux villes a joué (et joue encore) un rôle essentiel. Freiberg, avec ses 46000 habitants, est une ville bourgeoise, qui abrite une petite université et qui s'enorgueillit de posséder l'un des plus beaux théâtres de Saxe (une réplique en miniature du célèbre Semperoper de Dresde). Döbeln, qui ne compte que 23000 habitants, n'a pas les mêmes traditions théâtrales et a été sévèrement touchée par la crise économique qui sévit au sein des nouveaux Länder. À cela s'ajoute que Freiberg se situe dans la zone d'influence de Dresde, ville d'art et de culture, tandis que Döbeln est plus proche de Leipzig, ville commerçante.

«Döbeln fait partie de la zone d'influence de Leipzig, très nettement. [...] Les gens de Freiberg au contraire se sentent proches de Dresde. Dresde est par tradition une ville de culture. [...] Il y a toujours eu des villes de théâtre en RDA, c'est ainsi. Freiberg et Dresde sont des villes avec une riche tradition théâtrale à l'Est, comme Meiningen ou Weimar. Il $\mathrm{y}$ a des villes de théâtre et il y a les autres, qui n'en sont pas». Comédien, Mittelsächsiches Theater, Freiberg, décembre 2001.

Et dans tous les cas, les négociations sont mouvementées, illustrées par des revirements, des jeux d'alliance et de contre-alliance, qui font intervenir les intendances des différents théâtres, les représentants des municipalités, mais aussi plus directement 
les habitants. La fusion qui a lieu en 2001 entre Plauen et Zwickau témoigne bien de ce processus complexe. En effet, dès 1993, l'intendant de Zwickau évoque l'idée d'un Westsächsiches Landestheater, qui réunirait les théâtres de Zwickau, Plauen et Annaberg. Mais Plauen et Annaberg refusent, par crainte de s'engager dans un processus qui serait dominé par Zwickau. En 1996, la municipalité de Zwickau reprend l'initiative. Mais entre-temps Plauen a ouvert des négociations avec Hof, une petite ville de Bavière (9). La distance entre Plauen et Hof est inférieure à celle qui sépare Plauen de Zwickau et par ailleurs Hof ne compte que 51000 habitants (contre 104000 pour Zwickau), ce qui rassure les représentants de Plauen (70 000 habitants). Mais les habitants de Hof se mobilisent contre le projet en créant une initiative citoyenne (Bürgerinitiative): brandissant la menace d'un boycott, ils parviennent à faire reculer leurs représentants. Devant ces multiples tergiversations, le ministère de la Culture de la Saxe lance finalement un ultimatum à Plauen et Zwickau et contraint les deux villes à une fusion rapide (le contrat est signé en 1998 et la fusion est effective en 2000). Tout en étant un acteur essentiel de la rationalisation du paysage théâtral public, le Land est ici soucieux de se placer au-dessus des conflits engendrés par les processus de fusion.

«Dans le cas de la fusion entre Plauen et Zwickau, [...] cela s'est finalement terminé dans mon bureau et là, il a fallu déployer beaucoup de patience pour ménager les susceptibilités. [...] Parfois, c'est ainsi, malgré la nécessité évidente de la fusion, nécessité financière, cette nécessité pour les théâtres n'est pas reconnue ou même niée. C'est un problème que nous n'avons pas encore résolu». Ministre de la Culture du Land de la Saxe, Dresde, avril 2001.

\section{Des fusions rarement équilibrées}

Les craintes qui se manifestent à travers ces jeux d'alliance sont d'autant plus compréhensibles qu'une fusion est rarement équilibrée, et fait intervenir des partenaires inégaux. Même avec un partage des genres, l'une des deux villes s'impose toujours comme le centre de gravité de la nouvelle entité. La localisation de la nouvelle intendance est d'ailleurs un enjeu fort au moment des négociations. La ville qui compte le plus d'habitants est généralement en position dominante (par exemple Gera, Eisenach, Zwickau et Freiberg dans les cas qui nous intéressent).

Mais c'est surtout dans la répartition des postes entre les deux ensembles que le caractère inégalitaire de la fusion se manifeste. En effet, la fusion des ensembles s'accompagne généralement d'une réduction globale des effectifs et il est souvent

(9) Plauen se situe dans le Vogtland, une zone de la Saxe qui est frontalière de la Bavière. facile de distinguer parmi les deux ensembles d'origine qui en ressort gagnant. Ainsi, pour le théâtre d'Altenburg-Gera, l'Ensemble de Gera se maintient à $67 \%$ après la fusion, celui d'Altenburg à $45 \%$ seulement. Gera, qui conserve le Musiktheater, voit ses solistes relativement préservés (douze chanteurs sur dix-neuf et dix chanteuses sur treize restent dans le nouvel ensemble), tandis qu'Altenburg doit se séparer de sept de ses douze chanteurs et de sept de ses dix chanteuses. Mais la contrepartie n'est pas tout à fait vraie pour le Schauspiel (qui revient à Altenburg) : certes, Altenburg sauve une partie de ses comédiens (cinq hommes sur douze et six femmes sur dix), mais Gera garde douze de ses seize comédiens et cinq de ses dix comédiennes.

\section{Les conséquences organisationnelles des fusions}

Deux enjeux importants se dessinent lorsque l'on considère les conséquences à court et moyen terme des fusions sur les organisations théâtrales. Le premier concerne la mise en œuvre du travail théâtral et les contraintes nouvelles qui émergent, pour l'institution et pour ses personnels, en raison de la multiplication des lieux scéniques. Le second est lié à l'ancrage traditionnel des théâtres publics allemands dans des villes et des histoires culturelles locales. La fusion, notamment parce qu'elle brouille les mécanismes d'identification qui unissent l'institution à son public, vient ici encore contraindre l'organisation de la production théâtrale.

\section{La recomposition du travail théâtral sous de nouvelles contraintes}

« Les hommes politiques croient souvent de façon un peu rapide que la simple addition de six petits orchestres en engendre un grand très bon. C'est une idée idiote, une idée de comptable ». August Everding, président du Deutsche Bühnenverein, in Theater der Zeit, 11/1991, p. 44.

Comment à partir de plusieurs théâtres n'en faire qu'un? Comment gérer les nouvelles contraintes organisationnelles nées de ce rapprochement et de la multiplication des lieux scéniques ? Ainsi peut se résumer le problème de la fusion des Ensembles, tel qu'il se pose dans le cadre du Stadttheater allemand. En effet, comme le souligne un éditorial de la $G D B A(10)$, « la réunion de deux théâtres existants n'accroît pas nécessairement l'efficacité artistique. $\mathrm{Au}$ contraire : les contraintes supplémentaires que l'on fait peser sur les personnes à travers l'obliga-

(10) Genossenschaft Deutscher Bühnen Angehöriger, syndicat créé en 1871 et qui représente les membres des professions artistiques du théâtre en Allemagne fédérale. À l'heure actuelle, cette organisation regroupe environ 7000 adhérents. 
tion de se déplacer ont des conséquences sur leur travail et sur les productions. Les temps de répétition pour travailler les œuvres se raccourcissent et sont de plus en plus compliqués à organiser. Du côté de la technique, il est nécessaire de créer des possibilités de déplacement. Les transports spéciaux pour les décors, les bus pour le personnel, tout ce qui doit être déplacé entraîne des coûts supplémentaires » (Herdelin, 1993). Plusieurs éléments doivent donc être pris en compte pour évaluer l'impact des fusions sur l'activité théâtrale des Ensembles : la distance entre les lieux scéniques, les contraintes techniques et les modes de réorganisation du travail théâtral.

Les fusions ont généralement lieu entre des villes assez proches (une trentaine de kilomètres), mais on trouve des exceptions, puisque Rudolstadt et Eisenach sont distantes de 90 kilomètres. Les difficultés liées au transport des décors et à leur démontage sont toutefois communément partagées, et ce d'autant plus que les théâtres des nouveaux Länder restent, après la fusion, des théâtres de répertoire : les spectacles sont présentés en alternance durant toute la saison dans les différents lieux scéniques. Diverses stratégies sont néanmoins mises en œuvre pour tenter de minimiser les déplacements de décors d'une ville à l'autre. Par exemple, une pièce jouée en alternance dans une ville pendant la première moitié du mois se déplacera ensuite dans l'autre ville pour la deuxième quinzaine (la rotation peut aussi se faire sur le principe d'un mois sur deux, voir même d'un semestre sur deux). Mais ces formes de stabilisation sont toutes relatives. Pour certaines pièces, une rotation rapide est impérative. C'est le cas notamment entre novembre et janvier pour les contes de Noël, qui doivent être représentés simultanément dans les deux villes, ce qui accroît la charge de travail des équipes techniques. À cela s'ajoute le problème de la compatibilité des scènes sur lesquelles l'ensemble se produit en alternance. De coûteux travaux sont parfois nécessaires pour permettre l'alignement des espaces scéniques sur des normes communes.

Pour les comédiens (ou les chanteurs), la fusion entraîne de nouvelles conditions de travail et une nouvelle gestion des ressources humaines. En effet, les fusions imposent aux membres des ensembles une forte mobilité, qu'ils doivent souvent gérer par eux-mêmes (les théâtres n'organisent que rarement des transports collectifs et les membres des ensembles doivent alors prendre en charge leurs déplacements dans le cadre des répétitions et des représentations). La réorganisation des emplois du temps, générée par la multiplication des lieux scéniques, est donc une contrainte forte pour les membres des ensembles, même si la présence de plusieurs lieux scéniques permet aux comédiens de jouer une même pièce un plus grand nombre de fois et plus longtemps.
«Le transport des comédiens de chez eux aux différents lieux de répétition a été à lui tout seul une exigence très forte et a engendré les plus grandes difficultés. La plupart de ceux qui ont demandé des ruptures de contrat volontaires [...] l'ont fait à cause de ce problème. On a essayé au départ de mettre en place des dédommagements financiers, mais on s'est vite rendu compte que ce n'était pas faisable. L'intendant a alors décidé que des transports seraient organisés pour les répétitions générales et pour les premières. Pour toutes les autres représentations, il faut se débrouiller tout seul». Responsable des relations au public, théâtre de Plauen-Zwickau, Zwickau, décembre 2001.

«Pour nous la fusion signifiait donc [...] que nous allions aussi jouer à Döbeln et que les deux maisons seraient donc nos lieux de travail. Cela implique bien sûr des contraintes, parce que nous sommes responsables de nos déplacements, que nous effectuons avec notre voiture privée. [...] C'est contraignant, parce qu'il arrive souvent que l'on ait une répétition tôt ici à Freiberg, puis encore un petit quelque chose dans l'après-midi, et que le soir nous jouions à Döbeln, et ensuite il faut que nous rentrions chez nous [à Freiberg]. Donc c'est une charge». Comédienne, Mittelsächsiches Theater, Freiberg, décembre 2001.

«Par exemple, nous pouvons avoir deux répétitions à Altenburg dans la même journée, la première de 10 heures à 14 heures, et la seconde en fin d'aprèsmidi. Mais que peut-on faire là-bas dans l'intervalle [il habite à Gera] ? Le temps de trajet, c'est une chose qui n'est finalement pas si grave. Par contre, ensuite, on est coincé là-bas». Comédien, théâtre d'Altenburg-Gera, Gera, avril 2002.

En interne, la fusion est donc vécue comme un pis-aller qui certes garantit pour un temps la survie du théâtre, mais qui n'est accepté que faute de mieux et qui est vécu sous le mode de la contrainte. Ces réticences s'observent lors des négociations préalables aux fusions, qui s'accompagnent souvent de manifestations ou de pétitions organisées par les Ensembles concernés. Mais surtout les fusions entraînent parfois des scissions volontaires. Ainsi, au moment de la fusion entre Eisenach et Rudolstadt (Eisenach conserve le Musiktheater et Rudolstadt le Schauspiel), qui se caractérise, rappelons-le, par la grande distance qui sépare les deux villes, des comédiens ont refusé le transfert du Schauspiel à Rudolstadt et ont choisi de créer leur propre troupe, sous un statut associatif, pour continuer de jouer à Eisenach.

\section{Les enjeux de l'ancrage local}

Une caractéristique importante du Stadttheater allemand est d'être ancré dans un environnement local et d'adapter son offre à un public donné. Des difficultés surgissent donc dès qu'il s'agit d'élaborer une même programmation pour deux villes 
dont les caractéristiques sociales et culturelles sont parfois très différentes. Par ailleurs, les rancœurs provoquées par le processus de fusion ont pu, dans certains cas, attiser les tensions. Les comédiens sont très sensibles à ce problème et l'évoquent fréquemment en situation d'entretien.

«À Döbeln, nous ne trouvons pas une grande résonance. Si l'on considère le potentiel de spectateurs, c'est une ville qui n'a pas d'écoles supérieures, pas d'universités, pas d'entreprises. [...] Nous avons peu de spectateurs là-bas, et c'est dommage, parce que nous proposons la même qualité. [...] Nous ne jouons pas autre chose là-bas, il n'y a pas un deuxième répertoire. [...] J'ai fait " $x$ " mises en scène à Döbeln, et ça a toujours été extraordinairement difficile de faire quelque chose avec le public. Ce qui ici [à Freiberg] a du succès, ça tombe à plat là-bas, ça part en fumée, et c'est resté ainsi, c'est compliqué». Comédien, Mittelsächsiches Theater, Freiberg, décembre 2001.

«Un exemple: nous avons joué Kabale und Liebe, ça a été la première pièce qu'on a jouée à Altenburg après la fusion [suite au partage des genres, les comédiens sont installés à Gera]. Et au premier rang, il y avait tous les membres de l'association des amis du théâtre d'Altenburg, et de façon démonstrative, ils n'ont pas applaudi. C'est quelque chose qui ne s'oublie pas. Et aujourd'hui encore, on sent des tensions ». Comédien, théâtre d'AltenburgGera, Gera, avril 2002.

Pour éviter que l'une des deux villes (et donc l'un des deux publics) ne se sente lésée par la fusion, les intendances tentent de jouer sur la répartition des premières et des représentations entre les différents lieux scéniques.

«Nous faisons vraiment des efforts désespérés depuis six ans, pour expliquer à Döbeln et à ses habitants qu'ils sont pour nous tout aussi importants [que Freiberg]. Nous essayons de faire quelques premières à Döbeln, nous appelons chacune des premières représentations premières. Et nous avons aussi essayé d'organiser des fêtes autour des premières dans le théâtre [...] mais les gens de Döbeln ne viennent pas. [...] Nous avons même conçu des affiches spéciales pour Döbeln [pour dissimuler l'asymétrie des représentations]». Chef dramaturge, Mittelsächsisches Theater, Freiberg, avril 2001.

Dans le cas de Plauen-Zwickau, l'alternance des premières est prévue par le contrat de fusion : « Dans les deux lieux scéniques, les mises en scène prévues par la programmation devront être réalisées. Les premières auront lieu alternativement à Zwickau et à Plauen. Cela vaut pour le Schauspiel comme pour le Musiktheater. Pour les concerts, des premières seront organisées en alternance. Ainsi, le public des premières doit se voir garantir, dans les deux villes, l'accès au spectre complet de la production $»(11)$. Pourtant, si l'on regarde par exemple les chiffres de la saison 2001-2002, on trouve sept premières à Plauen (dont une uniquement à Plauen) et cinq à Zwickau (dont deux uniquement à Zwickau). L'avantage donné par l'intendance à Plauen (qui craignait d'être le parent pauvre de la fusion), éveille le ressentiment du public de Zwickau, qui dénonce volontiers les faveurs accordées à Plauen.

En dehors des premières, la répartition des représentations présente souvent les mêmes déséquilibres. Ainsi dans le cas d'Altenburg et de Gera, le déséquilibre est assez marqué : entre 1995 et 2000 , on a en moyenne (toutes salles confondues) 776 représentations par an à Gera et seulement 456 à Altenburg. Toutefois, ici, l'écart observé est d'abord lié à la taille très différente des deux villes (qui se traduit en termes de public potentiel). Contrairement aux apparences, la fusion est d'ailleurs loin d'être défavorable à Altenburg puisqu'en 1994, avant la fusion, la ville n'accueillait que 300 représentations.

\section{Quel impact à moyen/long terme ?}

Enfin, au-delà des conséquences organisationnelles des fusions théâtrales qui participent à un mouvement plus large de rationalisation des institutions culturelles, on peut s'interroger sur l'hypothèse d'un effet retour de la réunification. Si le paysage théâtral des nouveaux Länder semble s'être progressivement adapté aux exigences structurelles de la réunification, les difficultés rencontrées - et qui sont souvent exacerbées dans le cas des fusions - ne conduisent-elles pas à fragiliser les principes mêmes du fédéralisme culturel ?

\section{Le cercle sans fin des restrictions}

La fusion est toujours présentée par les responsables de politiques culturelles des Länder comme un moyen de stabiliser les coûts de production tout en maintenant le niveau artistique (selon l'hypothèse qu'en l'absence de fusion les restrictions budgétaires imposées aux théâtres risquent de nuire au niveau moyen de leurs productions). La fusion est donc vue comme une forme de rationalisation des restrictions. Pour autant, la fusion se révèle toujours $a$ posteriori comme le point de départ et non pas comme l'aboutissement d'un processus de restriction.

Le cas d'Altenburg-Gera, une fusion qui intervient en 1995, est ici exemplaire. En effet, dans les années qui précèdent (1990-1994), les deux théâtres ont connu de fortes restrictions budgétaires, qui se sont traduites par des réductions de postes (de 285

(11) Grundlagenvertrag zwischen den Städten Zwickau und Plauen zur Fusion beider Theater, p. 2, version du 10 août 1998. 
à 224 à Altenburg et de 320 à 298 à Gera). Ces dernières touchent principalement l'administration et l'entretien, qui est privatisé. Mais entre 1995 et 1999, 79 autres postes ont été supprimés, faisant passer les effectifs du nouvel ensemble issu de la fusion de 534 à 455 . Et cette fois, ces réductions touchent aussi les professions artistiques. Puis pendant la saison 2000-2001, le budget du théâtre est réduit de 40 à 32 millions de $\mathrm{DM}$, ce qui entraîne brutalement cent nouvelles suppressions de postes et provoque la démission de l'intendant qui avait conduit la fusion. Les départements techniques et administratifs perdent alors 30 à $60 \%$ de leur personnel. Parmi les professions artistiques, quatorze postes sont supprimés pour le théâtre, deux pour le ballet et cinq pour l'opéra. Parallèlement, la nouvelle intendance diminue d'un tiers le nombre de représentations, puisque le manque de personnel technique rend désormais difficile la mise en place de représentations en parallèle dans les différents lieux scéniques.

\section{Les négociations de Haustarif}

Qui plus est, les restructurations postérieures aux fusions s'accompagnent fréquemment de l'adoption d'un Haustarif, une grille de salaire spécifique qui s'applique à un théâtre et par laquelle certaines catégories de personnels renoncent à des augmentations de salaire, voire même acceptent des rémunérations inférieures aux normes en vigueur dans les théâtres publics(12). Depuis 1994, pas moins de soixantedix accords de Haustarif (Haustarifverträge) ont été signés au niveau local, dont la grande majorité dans des théâtres des nouveaux Länder. Les entités fusionnées sont ici en première ligne.

Ainsi, au sein du Mittelsächsiches Theater, comme à Altenburg/Gera, un Haustarif a été mis en place après la fusion pour garantir (au moins provisoirement) le maintien des postes.

«L'accord d'établissement sur les salaires a pour origine le manque d'argent. Ceux qui nous financent, les pouvoirs publics, ne donnent pas plus d'argent que ce qu'ils donnaient auparavant, pas moins non plus ; mais les coûts, et en particulier les coûts sala-

(12) En Allemagne, il n'existe pas comme en France de salaire minimum fixé par l'État. Les normes salariales sont les résultats d'accords entre les partenaires sociaux. C'est le cas pour le théâtre public, qui dispose, au sein du NV Bühne (Normal Vertrag Bühne, le contrat qui encadre les activités artistiques dans les ensembles publics) d'un Tarifvertrag (accord salarial). Ce dernier est négocié par le Deutsche Bühnenverein (qui réunit les intendants et se situe donc du côté des employeurs) et par les principaux syndicats (la GDBA, mais aussi la Vereinigung Deutscher Opernchöre, qui représente les intérêts des choristes et la Gewerkschaft Deutscher Orchestervereinigung, qui regroupe les musiciens d'orchestre). Récemment révisé au 1er janvier 2003, le $N V$ Bühne fixe un salaire minimum mensuel de 1550 euros. riaux augmentent(13). Et pour sortir de cet écueil, l'ensemble a proposé de renoncer à une partie de son revenu dans les prochaines années, à $13 \%$, et en contrepartie il y a une sécurité de l'emploi. Les collègues ont renoncé à de l'argent, parce que nous avons tous pensé : si nous n'y renonçons pas, vingt ou vingt-cinq postes vont disparaître et si ce théâtre voit ses postes diminuer, cela va forcément jouer sur son potentiel artistique, et un mauvais théâtre est plus facile à éliminer de la carte qu'un théâtre qui a une bonne réputation dans la Saxe. C'est pour cela que les collègues ont pris cette décision, il y a eu un vote et $80 \%$ étaient pour. [...] Mais les salaires ici sont très bas et renoncer à $13 \%$ a été douloureux. Mais d'un autre côté, nous avons ainsi acheté le maintien de postes pour quelques années». Chef dramaturge, Mittelsächsiches Theater, Freiberg, avril 2001.

Si son organisation a entériné localement nombre de ces accords, en partenariat avec les syndicats et les municipalités de tutelle des théâtres concernés, le directeur général du Deutsche Bühnenverein, Rolf Bolwin, n'en souligne pas moins le danger à moyen terme de ces grilles tarifaires spécifiques: «Ces grilles salariales maison se retrouvent pour l'essentiel dans les nouveaux Länder. [...] Elles ont été pensées comme des solutions transitoires. [...] Mais on a constaté que cela ne suffirait pas et que la durée envisagée était trop courte. Pourtant, le fait que les théâtres des nouveaux Länder sortent du système de relations professionnelles en vigueur n'est certainement pas une solution. Les théâtres ont besoin de règles précises du côté de l'employé, pour pouvoir travailler sans conflit. De plus, il ne doit pas y avoir dans ces théâtres et ces orchestres des grilles salariales qui, sur la durée, seraient plus faibles que dans les anciens Bundesländer, c'est-à-dire qu'il ne doit pas y avoir de zone tarifaire de l'Est». Rolf Bolwin, mdr online, 27 août 2001.

\section{L'effet retour de la réunification?}

Les craintes exprimées en 2001 par Rolf Bolwin semblent bien s'être concrétisées et apparaissent même relativement modestes au vu des évolutions observées depuis le début de la décennie. En effet, non seulement les théâtres de l'Est ont adopté durablement des grilles tarifaires inférieures aux normes en vigueur au sein de la République fédérale, mais on observe clairement une extension des cas de Haustarifverträge qui va des petits aux grands théâtres et surtout des nouveaux aux anciens Länder. Le mouvement qui s'est amorcé à l'Est a donc fait tâche d'huile. Après avoir touché les scènes les plus prestigieuses des nouveaux Länder (notamment le

(13) Depuis 1990, les salaires de l'Est rattrapent progressivement les salaires de l'Ouest, ce qui entraîne de fait une augmentation de la masse salariale dans les théâtres. Les pouvoirs publics prônent donc des réductions d'effectifs pour contrer cette hausse. 


\section{Encadré 4}

\section{Un usage préventif du Haustarif qui fait débat : le Weimarer Modell (modèle de Weimar)}

Les projets de fusion lancés par le Land de Thuringe afin de restructurer son paysage théâtral n'épargnent pas la capitale du Land (Erfurt, 213000 habitants) et sa prestigieuse voisine (Weimar, 62000 habitants), distantes de $25 \mathrm{~km}$. On assiste durant l'année 2001 à une véritable lutte d'influence entre les deux villes. La municipalité et les habitants de Weimar, qui occupe une position unique dans l'histoire et dans l'imaginaire culturel allemand, s'opposent farouchement à l'idée d'un rapprochement avec Erfurt et d'une séparation des genres. Le Deutsche Bühnenverein publie une étude de faisabilité qui évalue positivement les retombées potentielles de la fusion (1) et recommande la création d'une "Theatergemeinschaft» (communauté de théâtres) associant, sous une forme de $\mathrm{GmbH}$, trois tutelles publiques (les villes d'Erfurt et de Weimar ainsi que le Land de Thuringe). Mais les initiatives citoyennes se multiplient et le conseil municipal de Weimar rejette définitivement le projet du Land en février 2002. L'intendant du Deutsches Nationaltheater (DNT) Weimar, Stefan Märki, est chargé par la ville d'élaborer des propositions alternatives. Avec l'aide d'un avocat berlinois, Peter Rau, il propose une forme particulièrement radicale de Haustarif.

Si les arguments avancés sont classiques (faire face au poids croissant des salaires dans le budget du théâtre et tenter de maintenir le nombre de postes - et donc la qualité artistique de l'Ensemble malgré la baisse des subventions publiques), l'initiative de S. Märki se distingue des autres cas évoqués dans cet article. En effet, elle ne s'inscrit pas dans le cercle vicieux de restrictions provoqué par une fusion (qui n'a pas lieu), mais intervient en amont pour tenter de proposer une alternative à la restructuration du paysage théâtral. C'est sans doute cette singularité, couplée au prestige de l'Ensemble concerné, qui confère au cas de Weimar sa position spécifique dans le débat sur l'avenir du système théâtral des nouveaux Länder, et plus largement de l'Allemagne réunifiée.

De manière assez classique, les membres de l'Ensemble renoncent à leur 13e mois et à toute augmentation jusqu'en 2008. En contrepartie, l'intendance s'engage à bloquer les licenciements et à lutter sans relâche contre toute tentative de fusion. Mais plus qu'un ajustement temporaire, le modèle de Weimar se veut « un premier pas sur le chemin conduisant à de nouvelles structures pour les théâtres municipaux allemands» (2). En changeant de statut juridique pour devenir une $\mathrm{GmbH}$ à laquelle la municipalité n'est pas associée (3), le DNT impose à ses membres de nouveaux contrats de travail (4) et pose directement la question d'une « privatisation » de la culture. En effet, ce faisant le théâtre n'est plus soumis aux grilles tarifaires en vigueur dans le service public allemand (pour ce qui concerne les professions non artistiques) ni aux conventions négociées par les syndicats et le Bühnenverein (en ce qui concerne les professions artistiques).

Les débats suscités par ce changement de statut tendent à cristalliser les positions des différents acteurs sociaux concernés. Du point de vue de l'intendant et de l'avocat P. Rau qui a activement participé à la conception du modèle, il s'agit de sortir d'une logique de fusions qui ne fait que « cimenter les problèmes structurels existants et qui s'avère contreproductive pour le théâtre » (5). Pour cela, il faut selon eux autonomiser les théâtres et les dégager des pièges d'une organisation tarifaire contraignante et peu adaptée aux exigences concrètes du travail théâtral. Face à ces discours qui remettent en cause les fondements mêmes du droit du travail allemand (et pas seulement du droit du travail théâtral), les différents syndicats sont plus ou moins virulents. Ver.di, qui représente les professions non artistiques du théâtre (6), dénonce une américanisation de la culture et une « fuite » en dehors de toute règlementation qui pourrait s'étendre à d'autres secteurs d'activité. La GDBA, principal syndicat réunissant les professions artistiques du théâtre, est plus modérée et reproche à ver. di d'aiguiser les tensions entre catégories de personnel au sein du théâtre, sans prendre la mesure de la crise qui menace le DNT. Hostile à la généralisation du modèle de Weimar, la GDBA craint en effet avant tout la fermeture du théâtre et a déjà entériné, quand elles semblaient inévitables, des adaptations locales, temporaires et négociées sous forme de Haustarife. Enfin le Bühnenverein, considéré comme un « dinosaure » par S. Märki qui ne lui pardonne pas d'avoir soutenu le projet de fusion avec Erfurt, tente d'apaiser les tensions et ce d'autant plus que l'affaire de Weimar intervient quelques mois à peine avant l'entrée en vigueur, au 1er janvier 2003, du nouveau contrat de soliste (Normal Vertrag Bühne) qui représente pour le Bühnenverein l'aboutissement de 10 ans d'effort et de négociations avec les syndicats représentant les professions artistiques. Rolf Bolwin prend ainsi le parti de neutraliser l'initiative de S. Märki en la resituant dans le contexte des nombreux Haustarife signés dans d'autres théâtres, notamment est-allemands, avec l'accord de son organisation : « En pratique, ce soi-disant modèle repose en fait sur une série de mesures d'économies budgétaires déjà proposées ailleurs, comme par exemple le renoncement aux congés payés et au 13e mois, que nous avons souvent mis en place avec l'accord des syndicats dans des théâtres et des orchestres des nouveaux Länder»(7). Mais dans les faits, la rupture est bien plus radicale et le DNT, en se détachant des autorités publiques et en renonçant à appartenir au Bühnenverein, affirme clairement sa position singulière au sein du paysage théâtral allemand.

(1) Machbarkeitsstudie zur Umsetzung der Empfehlungen der Sachverständigenkommission "Theater und Orchester in Thuringen" (EverdingKommission) aus dem Jahre 1996 - Die Theatergemeinschaft des Theaters Erfurt und des Deutschen Nationaltheaters Weimar", Deutsche Bühnenverein, Cologne, 2001.

(2) Site Internet du DNT, septembre 2006. On peut noter que cette formulation témoigne d'une évolution nette des positions de l'intendant S. Märki qui, en 2002/2003 au moment de la mise en œuvre du changement de statut de son institution, refusait le terme de «modèle» et insistait avant tout sur les conditions sociales, politiques et historiques propres à la ville de Weimar. Depuis, la médiatisation de la stratégie adoptée par le DNT et l'aggravation des problèmes financiers rencontrés par les théâtres allemands, dans les anciens comme dans les nouveaux Länder, ont donné un poids beaucoup plus fort à son initiative, qui est par exemple largement discutée au sein des différents partis politiques.

3) Notons qu'au début des années 1000 , environ $25 \%$ des théâtres publics allemands sont des GmbH. Mais ils continuent à être gérés par des tutelles publiques. Ce n'est plus le cas du DNT, qui devient une GmbH gérée par des personnes privées. Le seul antécédent était le cas du Berliner Ensemble, dont le dossier avait déjà été suivi par l'avocat P. Rau. La municipalité de Weimar, qui soutient ce changement de statut, continue néanmoins à subventionner le DNT et s'engage même à maintenir le niveau de ses subventions jusqu'en 2008.

(4) Dans les faits, les contrats antérieurs restent valables pendant un an. Passé ce délai, de nouveaux contrats sont signés au nom de la GmbH, que les salariés de l'Ensemble ont la possibilité de refuser. Théoriquement, ils restent alors employés par la ville. Mais cette dernière peut les licencier si elle prouve qu'elle n'est pas à même de leur fournir un autre emploi correspondant à leurs compétences. La ville de Weimar ne comptant qu'un seul théâtre, cette clause est extrêmement contraignante pour les membres de l'Ensemble qui seraient tentés de refuser le contrat que leur proposera la nouvelle $\mathrm{GmbH}$.

(5) S. Märki in Neue Musikzeitung, 2002/2003, p. 3.

(6) Relevant du service public du service public (au contraire des professions artistiques qui dépendent d'un contrat spécifique, le Normal Vertrag Bühne), ce sont elles qui ont le plus à perdre dans le changement de forme juridique de leur institution.

(7) R. Bolwin, in Theaterheute, août 2002, p. 1. 
Semperoper de Dresde), il s'étend désormais dans les anciens Länder où les théâtres du SchleswigHolstein et de Saarbrück sont aujourd'hui en première ligne.

De manière intéressante, et toutes choses égales par ailleurs, nous retrouvons ici une évolution mise en évidence dans d'autres domaines d'activité. Olivier Giraud et Michel LaLlEment (2001) soulignent ainsi qu'une reconfiguration des relations professionnelles est en cours dans les nouveaux Länder, qui répond à un mouvement conjoint «de décentralisation et de différentiation des conventions collectives». La réunification s'est accompagnée, dans un premier temps, de la signature d'accords concernant le rattrapage des salaires de l'Est sur ceux de l'Ouest. Mais dans le monde des théâtres publics, comme ailleurs, le compromis s'est avéré difficilement tenable. Les difficultés rencontrées se sont traduites «par l'activation de solidarités d'entreprise, les objectifs prioritaires étant la survie de l'unité productrice et l'adaptation aux nouvelles donnes du marché, quitte à promouvoir [...] des conditions de travail difficiles» (GIRAuD, LALLEMENT, 1998). Les accords locaux signés à l'Est, dès lors qu'ils ont essaimé dans les anciens Länder, ont fini par irriter les Allemands de l'Ouest, qui ont reproché à leurs concitoyens de casser les accords de branche pour sauver localement des emplois.

Les positions des principaux acteurs ont alors sensiblement évolué. Ainsi la GDBA, pourtant signataire des accords de Haustarif au sein des théâtres de l'Est, s'inquiète désormais de ce qui devient progressivement une règle, et non plus une exception destinée à accompagner la période de transition à l'Est. Lors de son assemblée générale de 2005, elle interpelle ainsi le Bühnenverein et demande aux intendants de veiller, au sein de leurs théâtres respectifs, au maintien du Tarifvertrag et au respect des conditions du Normal Vertrag Bühne. Rappelant que le monde théâtral «n'est pas une zone de non droit », la GDBA souligne avec force le rôle structurant du Tarifvertrag comme « instrument de régulation des conditions de travail ». Un éditorial de la même année dénonce l'érosion généralisée du paysage théâtral allemand et l'irréversibilité des accords signés localement (HerdLeIn, 2005).

Au niveau de la branche, le syndicat majoritaire des artistes de théâtre opère donc un rappel à la règle et aux conventions régissant le monde du travail théâtral. Mais au cas par cas, ses représentants se retrouvent toujours aux côtés du Bühnenverein pour sauvegarder les emplois menacés, quitte à accepter des conditions de salaire moins avantageuses. Ils se heurtent, au sein des institutions théâtrales, au syndicat ver.di. (14) (Vereinte Dienstleistungsgewer $k s c h a f t$ ) qui représente notamment les intérêts des personnels techniques des théâtres et qui s'oppose farouchement à la mise en place des Haustarife. Les institutions théâtrales se trouvent donc de plus en plus fréquemment confrontées à des conflits entre personnels artistiques (encore majoritairement favorables aux Haustarife lorsqu'ils permettent de sauvegarder des emplois) et personnels techniques (hostiles à ces accords(15)).

De ce point de vue, l'intégration des nouveaux Länder au sein de la République fédérale semble bien conduire progressivement à la remise en cause des principes fondamentaux sur lesquels s'étaient construites les formes de régulation du travail en RFA (BAFOIL, 1996).

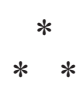

Le processus des fusions théâtrales qui s'est enclenché en Saxe et en Thuringe dans la première moitié des années 1990 ne semble pas encore être arrivé à son terme aujourd'hui. De nouvelles fusions se dessinent, qui impliquent des entités théâtrales déjà fusionnées, et qui parfois remettent en cause les fusions antérieures, témoignant ainsi de la complexité du processus de recomposition du paysage théâtral au sein des nouveaux Länder.

En effet, l'impact des fusions sur la stabilisation des coûts de fonctionnement des structures théâtrales publiques est difficile à évaluer. L'efficacité de cette stratégie de concentration du tissu théâtral reste donc incertaine, et ce d'autant plus que l'on ne dispose pas de statistiques fiables. La réticence des intendances et des ministères de la Culture à communiquer sur ces questions montre que les effets positifs espérés à moyen et long terme sont loin d'être évidents. Les enjeux des fusions au sein même des organisations théâtrales, leurs conséquences sur le travail au sein des ensembles, sont plus faciles à cerner. Ils mettent en évidence la force des interdépendances entre les politiques culturelles menées à l'échelle des Länder et les transformations organisationnelles qui affectent localement les structures concernées. Plus encore, les difficultés observées révèlent la fragilité du modèle théâtral hérité de la RDA et ses difficultés à affronter le changement de système. En ce sens, les fusions apparaissent

(14) Fondée en 2001 à Berlin, cette organisation est née du rapprochement de cinq syndicats présents dans le secteur des services. Elle a fait des politiques tarifaires son principal cheval de bataille.

(15) Rappelons que depuis la réunification, les départements techniques des théâtres, et c'est particulièrement vrai dans les cas de fusion, ont été les premiers touchés par les réductions de poste, qui sont intervenues plus tardivement et surtout moins massivement pour les professions artistiques. Les sacrifices déjà consentis localement par ces deux catégories au sein des ensembles sont donc très différents. 
bien pour le sociologue comme des cas extrêmes, qui cristallisent les problèmes rencontrés de façon générale par l'ensemble des théâtres publics des nouveaux Länder (VERDALLE (DE), 2003, 2006).

Mais dans le même temps, ces fusions remettent aussi en cause un modèle ouest-allemand qui sort fragilisé de son application à un tissu théâtral dont les caractéristiques étaient très éloignées de celles des anciens Länder. Loin d'exercer une influence à sens unique, la réunification pourrait donc bien conduire à une altération durable des principes fondateurs du fédéralisme ouest-allemand (Le GlOANNEC, 2001).

\section{Bibliographie}

BAFOIL F. (1996), « La crise des relations professionnelles en Allemagne de l'Est et l'évolution de la négociation paritaire allemande », in Politix, $\mathrm{n}^{\circ} 33$, pp. 87-110.

BARMEYER C. (2005), « Rapprochements des entreprises et rapprochements des hommes. Regard interculturel sur le leadership dans les coopérations franco-allemandes "), in Joly H. (sous la direction de), (2005), Formation des élites en France et en Allemagne, travaux et documents de CIRAC, Paris.

Giraud O., Lallement M., (1998), « Construction et épuisement du modèle néocorporatiste allemand. La réunification comme consécration d'un processus de fragmentation sociale », in Revue Française de Sociologie, n 43-1, pp. 39-69.

Giraud O., Lallement M., (2001), « L'État et les relations professionnelles en Allemagne: interactions et recompositions après la réunification ", in Le Gloannec A.-M. (sous la direction de), (2001), L'État en Allemagne. La République fédérale après la réunification, Presses de Sciences Po, Paris.

Herdlein H., (1993), « Einigungsvertrag verpflichtet kulturelle Substanz erhalten! », in Bühnengenosseschaft, $\mathrm{n}^{\circ} 5$, pp. 5-6.

Herdlein H. (2005), «Kulturelle Substanz erhalten - Arbeitslosigkeit im Entstehen bekämpfen », in Bühnengenosseschaft, ${ }^{\circ}$ 5, pp. 4-5.
Laborier P., (1992), «L'enjeu de la culture dans l'unification allemande. Du dirigisme centraliste au pluralisme démocratique ", in Allemagne d'aujourd'hui, n 121, pp. 146-165.

LABORIER P., (1996), "Conservation ou rénovation? Transitions de la politique culturelle », in Politix, $\mathrm{n}^{\circ} 33$, pp. 111-132.

Le Gloannec A.-M (sous la direction de), (2001), L'État en Allemagne. La République fédérale après la réunification, Presses de Sciences Po, Paris.

Verdalle L. (de) (sous la direction de), (2002), « D’une gestion centrale à une gestion locale de la culture : les théâtres au cœur de la transition est-allemand », in Nouvelle Alternative, $\mathrm{n}^{\circ}$ 57, pp. 12-22.

Verdalle L. (de), (2003), « La transition théâtrale dans l'ex-RDA: vers la recherche de nouvelles formes de régulation organisationnelle », in Sociologie du travail, vol. 45 , pp. 385-405.

Verdalle L. (de), (2006), Le théâtre en transition. De la RDA aux nouveaux Länder, collection « Dialogiques », éditions de la MSH, Paris.

WiesAnd A., (1992), « Vers le centralisme et la culture d'État? Sur le financement de la culture en Allemagne », Le Débat, $\mathrm{n}^{\circ} 70$, pp. 26-41. 